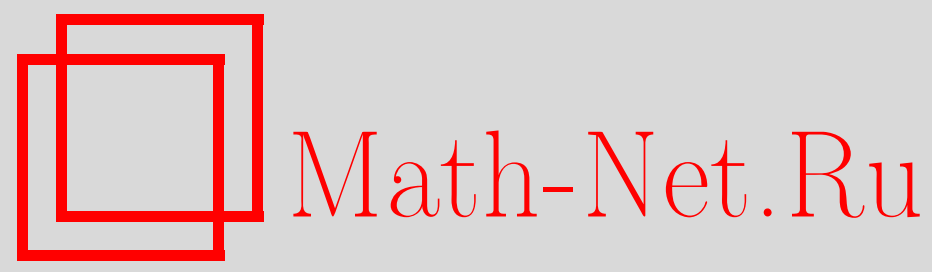

А. Хатамов, Многомерные аналоги прямых и обратных теорем Джексона и Бернштейна и их обобщения, Матем. заметки, 2000, том 67, выпуск 4, 608-615

DOI: https://doi.org/10.4213/mzm876

Использование Общероссийского математического портала Math-Net.Ru подразумевает, что вы прочитали и согласны с пользовательским соглашением http://www.mathnet.ru/rus/agreement

Параметры загрузки:

IP : 54.81 .137 .203

26 апреля 2023 г., 05:12:52

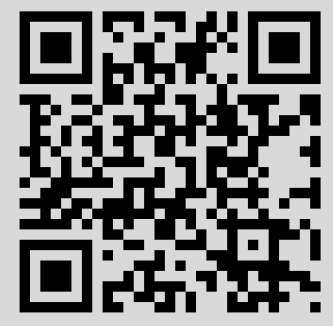




\section{МНОГОМЕРНЫЕ АНАЛОГИ ПРЯМЫХ И ОБРАТНЫХ ТЕОРЕМ ДЖЕКСОНА И БЕРНШТЕЙНА И ИХ ОБОБЩЕНИЯ}

\section{А. Хатамов}

Получен вариант многомерных аналогов классических прямых и обратных теорем Джексона и Бернштейна-Валле-Пуссена, более сильный, чем такие же аналоги, полученные С. М. Никольским, а также существенные обобщения этих теорем на полиномиальные приближения функций действительных переменных из пространств Никольского и Бесова на ограниченных областях с липшицевой границей. Прямая теорема, относящаяся к пространствам Никольского, ранее получена Ю. А. Брудным и обобщена на совместные полиномиальные приближения функций и их производных В.Н. Коноваловьм.

Библиография: 14 названий.

Пусть $\mathbb{R}^{n}-n$-мерное евклидово пространство с обычной нормой $\|\cdot\|(n \geqslant 1), G-$ открытое множество в $\mathbb{R}^{n}, L_{p}(G)$ - квазинормированное пространство всех измеримых действительнозначных функций на $G$, суммируемых в $p$-й степени с квазинормой

$$
\begin{aligned}
\|f\|_{p, G} & :=\left(\int_{G}|f(x)|^{p} d x\right)^{1 / p} \quad(0<p<\infty), \\
\|f\|_{\infty, G} & :=\sup \operatorname{vrai}\{|f(x)|: x \in G\} .
\end{aligned}
$$

При $0<p \leqslant \infty$ и целых $l \geqslant 0$ через $W_{p}^{(l)}(G)$ обозначим изотропное пространство Соболева функций $f \in L_{p}(G)$, имеющих на $G$ обобщенные в смысле Соболева частные производные $D^{(l)}(e) f\left(D^{(0)}(e) f \equiv f\right)$ порядка $l$ по направлению каждого $n$-мерного единичного вектора $e$ и обладающих конечной квазиполунормой (см. [1]):

$$
|f|_{W_{p}^{(l)}(G)}=\sup \left\{\left\|D^{(l)}(e) f\right\|_{p, G}: e \in \mathbb{R}^{n},\|e\|=1\right\} .
$$

При целых $k \geqslant 0$ символ $\Delta^{(k)}(\varepsilon e) f$ обозначает $k$-ю разность от функции $f$ с шагом $\varepsilon \geqslant 0$ по направлению единичного вектора $e$ :

$$
\begin{aligned}
\Delta^{(0)}(\varepsilon e) f(x) & \equiv f(x), \\
\Delta^{(k)}(\varepsilon e) f(x) & :=\sum_{i=0}^{k}(-1)^{k-i} C_{k}^{i} f(x+i \varepsilon e) \quad(k \geqslant 1) .
\end{aligned}
$$


Если для каждого $n$-мерного единичного вектора $e$ и некоторого целого числа $l \geqslant 0$ на множестве $G$ существует $D^{(l)}(e) f \in L_{p}(G)$, то при $\delta \geqslant 0$ определим изотропные модули гладкости $k$-го порядка для производных $l$-го порядка

$$
\omega_{k}^{(l)}(f, \delta)_{p, G}=\sup \left\{\sup \left\{\left\|\Delta^{(k)}(\varepsilon e) D^{(l)}(e) f\right\|_{p, G_{k \varepsilon e}}: 0 \leqslant \varepsilon \leqslant \delta\right\}: e \in \mathbb{R}^{n},\|e\|=1\right\}
$$

где $G_{k \varepsilon}-$ подмножество $G$, состояшее из всех точек $x \in G$, для которых отрезки прямой $[x, x+k \varepsilon e]$ полностью лежат в $G$. Верхний (нижний) индекс в обозначении $\omega_{k}^{(l)}(\cdot)$ мы будем опускать в случае его равенства нулю (единице). Пусть $\alpha>0$, целые числа $k$ и $\nu$ удовлетворяют неравенствам $0 \leqslant \nu<\alpha<k+\nu$. Такую пару чисел $(k, \nu)$ называют допустимой парой. При $0<p \leqslant \infty, 0<q \leqslant \infty$ через $B_{p, q}^{\alpha}(G)$ обозначим пространство Бесова (при $q=\infty$ - пространство Никольского $H_{p}^{\alpha}(G)=B_{p, \infty}^{\alpha}(G)$ ) функций $f \in L_{p}(G)$, для которых при каждом единичном векторе $e$ на множестве $G$ существует $D^{\nu}(e) f \in L_{p}(G)$ и конечна квазиполунорма

$$
\begin{aligned}
|f|_{B_{p, q}^{\alpha}(G)} & :=\left(\int_{0}^{\infty}\left[t^{-(\alpha-\nu)} \omega_{k}^{(\nu)}(f, t)_{p, G}\right]^{q} \frac{d t}{t}\right)^{1 / q} \quad \text { при } \quad 0<q<\infty, \\
|f|_{B_{p, \infty}^{\alpha}(G)}:=\sup \left\{t^{\nu-\alpha} \omega_{k}^{(\nu)}(f, t)_{p, G}: t>0\right\} & \text { при } \quad q=\infty .
\end{aligned}
$$

Как известно,

$$
\|f\|_{B_{p, q}^{\alpha}(G)}:=\|f\|_{p, G}+|f|_{B_{p, q}^{\alpha}(G)}
$$

при $1 \leqslant p \leqslant \infty$ и $1 \leqslant q \leqslant \infty$ является нормой пространства $B_{p, q}^{\alpha}(G)$ и превращает его в банахово пространство [1], а при $0<p \leqslant \infty$ и $0<q \leqslant \infty$ - является квазинормой пространства $B_{p, q}^{\alpha}(G)[2]$.

Для целых $N \geqslant 0$ и $n \geqslant 1$ через $P_{N, n}$ обозначим множество всех алгебраических полиномов $g(x)=\sum C_{\lambda} x^{\lambda}$ степени не вьше $N$ по совокупности $n$ переменных, где $\lambda=\left(\lambda_{1}, \ldots, \lambda_{n}\right)$ - мультииндекс с цельми неотрищательными компонентами $\lambda_{i}$, $x^{\lambda}=x_{1}^{\lambda_{1}}, \ldots, x_{n}^{\lambda_{n}}, C_{\lambda}$ - действительные числа, а суммирование производится по всем $\lambda$, для которых $|\lambda|:=\lambda_{1}+\cdots+\lambda_{n} \leqslant N$. Через $E_{N}(f, G)_{p}$ обозначим наименьшее уклонение функции $f \in L_{p}(G)$ от многочленов $g \in P_{N, n}$ по квазинорме пространства $L_{p}(G)$, т. e.

$$
E_{N}(f, G)_{p}:=\inf \left\{\|f-g\|_{p, G}: g \in P_{N, n}\right\} .
$$

Пусть $0<\alpha<\infty, 0<p \leqslant \infty, 0<q \leqslant \infty$. Пространства

$$
\begin{aligned}
E_{p, q}^{\alpha}(G):= & \left\{f \in L_{p}(G):\|f\|_{E_{p, q}^{\alpha}(G)}\right. \\
& \left.:=\|f\|_{p, G}+\left(\sum_{N=1}^{\infty}\left[N^{\alpha-1 / q} E_{N}(f, G)_{p}\right]^{q}\right)^{1 / q}<\infty\right\}, \\
E_{p, \infty}^{\alpha}(G):= & \left\{f \in L_{p}(G):\|f\|_{E_{p, \infty}^{\alpha}(G)}\right. \\
& \left.:=\|f\|_{p, G}+\sup \left\{N^{\alpha} E_{N}(f, G)_{p}: N=1,2, \ldots\right\}<\infty\right\}
\end{aligned}
$$

являются аппроксимационными пространствами, а величина $\|\cdot\|_{E_{p, q}^{\alpha}(G)}-$ квазинормой пространства $E_{p, q}^{\alpha}(G)[2]$. 
Пусть $A$ и $B$ - квазинормированные пространства. Если вьполнено теоретико-множественное включение $A \subset B$ и неравенство $\|a\|_{B} \leqslant C\|a\|_{A}$ для любого $a \in A$, где $C$ - положительная величина, не зависящая от $a$, то будем говорить, что $A$ вложено непрерывно в $B$ и пишем $A \hookrightarrow B$.

Пусть $W^{l} H^{\alpha}(E)$ - класс всех функций $f$, имеющих непрерьвную производную порядка $l \geqslant 0$ на некотором открытом или замкнутом множестве $E \subset \mathbb{R}$, для модуля непрерьвности которых при некоторых $K=$ const $>0,0<\alpha \leqslant 1$ и всех $\delta \geqslant 0$ справедлива оценка $\omega\left(f^{(l)}, \delta\right) \leqslant K \delta^{\alpha}$. Через $\widetilde{W^{l}} H^{\alpha}$ обозначим класс всех $2 \pi$-периодических функций $f \in W^{l} H^{\alpha}(\mathbb{R})$.

Всюду ниже $C\left(\beta_{1}, \beta_{2}, \ldots\right), C_{1}\left(\beta_{1}, \beta_{2}, \ldots\right), \ldots$ обозначают положительные величины, зависящие от параметров, указанных в скобках, и индексов.

Прямая теорема Джексона [3] и обратная теорема Бернштейна [4], уточненная Валле-Пуссеном [5], дают следующую характеристику класса $\widetilde{W}^{l} H^{\alpha}(l=0,1, \ldots, 0<$ $\alpha<1)$ : для того чтобы $f \in \widetilde{W}^{l} H^{\alpha}$, необходимо и достаточно выполнение неравенства

$$
E_{N}^{T}(f) \leqslant C(l) N^{-l-\alpha}
$$

где $E_{N}^{T}(f)$ - наилучшее приближение функции $f \in \widetilde{W^{l}} H^{\alpha}$ тригонометрическими полиномами степени не вьше $N$ в равномерной метрике.

В той же работе [3] доказано сохранение порядка стремления к нулю правой части оценки (1) для $E_{N}(f)$ - наилучших приближений функции $f \in W^{l} H^{\alpha}([-1,1])$ алгебраическими многочленами степени не выше $N$ на отрезке $[-1,1]$, в [4], [5] показано, что обратная теорема имеет место не для всего отрезка $[-1,1]$, а только на каждом отрезке $[a, b] \subset(-1,1)$, т. е. из оценки $(1)$ для $E_{N}(f)$ вытекает принадлежность $f$ классу $W^{l} H^{\alpha}([a, b])$ для любого отрезка $[a, b] \subset(-1,1)$ (см. также [6, с. 91]). Отметим также, что Никольским установлена [7] возможность характеризации пространства $H_{\infty}^{\alpha}(G)$ $(0<\alpha<\infty)$, где $G$ - ограниченная область с гладкой границей в $\mathbb{R}^{n}(n \geqslant 2)$, в терминах наилучших приближений алгебраическими многочленами на кубе $T$, содержащем $G$, функций $f \in H_{\infty}^{\alpha}(G)$, допускаюших продолжение до функций $f \in H_{\infty}^{\alpha}(T)$.

Такая же ситуация справедлива для функций многих действительных переменных пространств Никольского и Бесова, что следует из результатов монографии [1] Никольского, описываюших эти пространства в терминах наилучших приближений тригонометрическими полиномами и целыми функциями экспоненциального сферического типа, а также из следующей теоремы и предложения настоящей работы.

ТЕОремА. Если $G$-ограниченная область в $\mathbb{R}^{n}(n \geqslant 2)$ с липиицевой границей, то при $0<\alpha<\infty, 1 \leqslant p \leqslant \infty, 0<q \leqslant \infty$ имеем

$$
B_{p, q}^{\alpha}(G) \subset E_{p, q}^{\alpha}(G) \subset B_{p, q}^{\alpha}(D)
$$

әде $D$ - любое открытое мнохсество, компактно влохенное в $G$.

Нижеследующее предложение подтверждает невозможность замены множества $D$ на $G$ в приведенной теореме.

ПРЕДЛОЖЕНИЕ. Вложение

$$
E_{p, q}^{\alpha}\left((-1,1)^{n}\right) \subset B_{p, q}^{\alpha}\left((-1,1)^{n}\right)
$$


не имеет места ни при каких значениях параметров $\alpha, p, q$, удовлетворяющих какому-либо из следующих условий:

а) $1 / 2+1 / p<\alpha \leqslant 3 /\left(2 p_{1}\right)+1 / 2-\varepsilon$, где $\varepsilon>0$ произвольно мало, $p_{1}=\max \{1, p\}$, $2 / 3<p<\infty, 0<q \leqslant \infty($ при $q=\infty$ можно брать $\varepsilon=0)$;

б) $1 / 2<\alpha \leqslant 1-\varepsilon$, где $\varepsilon>0$ произвольно мало, $p=\infty, 0<q \leqslant \infty($ при $q=\infty$ мохсно полохить $\varepsilon=0$ ).

Приведенная выше теорема представляет собой более сильньй вариант многомерных аналогов классических прямых и обратных теорем Джексона и Бернштейна-ВаллеПуссена, чем такие же аналоги, полученные Никольским в [7], и их существенные обобщения на полиномиальные приближения функций многих действительных переменных из пространств Никольского и Бесова на ограниченных областях с липшицевой границей.

ЗАмЕчАниЕ. Прямая теорема, касающаяся пространств Никольского (левое вложение приведенной теоремы при $q=\infty$ ), ранее получена Брудным и обобщена на совместные полиномиальные приближения функций и их производных Коноваловым (см. ниже в начале $\S 1$ теорему А и оценки (2), (4) и (5)).

\section{§ 1. Доказательство теоремы}

1.1. Сначала докажем первое вложение теоремы. Для этого воспользуемся частным случаем теоремы Коновалова [8]. Прежде чем сформулировать эту теорему, приведем следующее определение.

ОПРЕДЕЛЕНИЕ. Величина $\omega_{k}^{(l)}(f, \delta)_{p}$ называется функиией тuпа $k$-го модуля непрерывности, если при каждом $\delta \geqslant 0$ она конечна, неотрицательна, не убьвает при возрастании $\delta$ и при любом $\tau \geqslant 0$ удовлетворяет неравенству

$$
\omega_{k}^{(l)}(f, \tau \delta)_{p} \leqslant(1+\tau)^{k} \omega_{k}^{(l)}(f, \delta)_{p}
$$

Теорема А (В.А. Коновалов). Если $G$ - ограниченная область в $\mathbb{R}^{n}(n \geqslant 2) c$ липиичевой граничей, $1 \leqslant p \leqslant \infty, l$ u $k$-челье неотричательные числа, причем $l+k>0$, и для функиии $f$, определенной на $G, \omega_{k}^{(l)}(f, \delta)_{p}-$ функиия типа $k$-го модуля непрерывности, то при каждом $N \geqslant l+k-1$ найдется полином $g \in P_{N, n}$ такой, что при $0 \leqslant s \leqslant l$ выполнено неравенство

$$
|f-g|_{W_{p}^{(s)}(G)} \leqslant C(n, l, k, s, G) \omega_{l-s+k}^{(s)}\left(f, \frac{1}{N}\right)_{p, G}
$$

Полагая в теореме А $s=l=0$ для любой функции $f \in L_{p}(G)$ при $1 \leqslant p \leqslant \infty$, $k=1,2, \ldots, N \geqslant k-1$ имеем оценку

$$
E_{N}(f, G)_{p} \leqslant C(n, k, G) \omega_{k}\left(f, \frac{1}{N}\right)_{p, G} .
$$

Эта оценка фактически установлена ранее Брудным [9], [10]. 
Пусть $f \in B_{p, q}^{\alpha}(G)$ - любая функция. Тогда $f \in L_{p}(G)$ и

$$
\begin{gathered}
\int_{0}^{\infty} t^{-1-\alpha q} \omega_{[\alpha]+1}(f, t)_{p, G} d t<\infty \quad \text { при } \quad 0<q<\infty, \\
\sup \left\{t^{-\alpha} \omega_{[\alpha]+1}(f, t)_{p, G}: t>0\right\}<\infty \quad \text { при } \quad q=\infty .
\end{gathered}
$$

Так как пространства $B_{p, q}^{\alpha}(G)$ для областей $G$ с липшищевой границей не зависят от допустимых пар $(k, \nu)$, то в их определении можно положить $k=[\alpha]+1$ и $\nu=0$. На основании этого, считая $q=\infty$, из (2) и (4) получим оценку

$$
\begin{aligned}
\sup \left\{N^{\alpha} E_{N}(f, G)_{p}: N=1,2, \ldots\right\} & \leqslant C(n, \alpha, G) \sup \left\{t^{-\alpha} \omega_{[\alpha]+1}(f, t)_{p, G}: t>0\right\} \\
& =C(n, \alpha, G)|f|_{B_{p, \infty}^{\alpha}}(G)
\end{aligned}
$$

Пусть теперь $0<q<\infty$. С учетом (2), (3) и определения квазиполунормы пространств $B_{p, q}^{\alpha}(G)$ получаем

$$
\begin{aligned}
|f|_{B_{p, q}^{\alpha}(G)}^{q} & \geqslant \int_{0}^{1} t^{-1-\alpha q} \omega_{[\alpha]+1}^{q}(f, t)_{p, G} d t=\int_{1}^{\infty} \tau^{-1+\alpha q} \omega_{[\alpha]+1}^{q}\left(f, \frac{1}{\tau}\right)_{p, G} d \tau \\
& =\sum_{N=1}^{\infty} \int_{N}^{N+1} \tau^{-1+\alpha q} \omega_{[\alpha]+1}^{q}\left(f, \frac{1}{\tau}\right)_{p, G} d \tau \\
& \geqslant C(\alpha, q) \sum_{N=1}^{\infty} N^{-1+\alpha q} \omega_{[\alpha]+1}^{q}\left(f, \frac{1}{N+1}\right)_{p, G} \\
& \geqslant 2^{-1-[\alpha]} C(\alpha, q) \sum_{N=1}^{\infty} N^{-1+\alpha q} \omega_{[\alpha]+1}^{q}\left(f, \frac{1}{N}\right)_{p, G} \\
& \geqslant C(\alpha, q, n, G) \sum_{N=1}^{\infty} N^{-1+\alpha q} E_{N}^{q}(f, G)_{p} .
\end{aligned}
$$

Из (6) по определению квазинорм пространств $E_{p, q}^{\alpha}(G)$ и $B_{p, q}^{\alpha}(G)$ получим неравенство

$$
\|f\|_{E_{p, q}^{\alpha}(G)} \leqslant C_{1}(\alpha, q, n, G)\|f\|_{B_{p, q}^{\alpha}(G)} .
$$

Итак, первое вложение теоремы доказано.

1.2. Докажем теперь второе вложение. Пусть вьполнены условия теоремы и $f \in$ $E_{p, q}^{\alpha}(G)$. Функцию $f$ представим в виде ряда, сходящегося к $f$ по норме $L_{p}(G): f(x)=$ $\sum_{l=0}^{\infty} u_{l}(x)$, где $u_{0}=g_{1}, u_{l}=g_{2^{l}}-g_{2^{l-1}}$ при $l=1,2, \ldots$, а $g_{2^{l}} \in P_{2^{l}, n}$ - полином наилучшего $L_{p}(G)$-приближения функции $f$ подпространством $P_{2^{l}, n}$. Легко заметить, что

$$
\left\|u_{0}\right\|_{p, G} \leqslant 2\|f\|_{p, G}, \quad\left\|u_{l}\right\|_{p, G} \leqslant 2 E_{2^{l-1}}(f, G)_{p} .
$$

В работе [11] Никольским доказана следующая оценка для $p_{N} \in P_{N, n}$ :

$$
\begin{aligned}
& \left\|\left(D^{\lambda} p_{N}(x)\right)\left(\sqrt{r(x, \partial G)}+N^{-1}\right)^{\gamma+|\lambda|}\right\|_{p, G} \\
& \quad \leqslant C(\lambda, \gamma, n, G) N^{|\lambda|}\left\|p_{N}(x)\left(\sqrt{r(x, \partial G)}+N^{-1}\right)^{\gamma}\right\|_{p, G},
\end{aligned}
$$


где $\lambda$ - целочисленный мультииндекс с неотрицательными компонентами $\lambda_{i}, D^{\lambda}:=$ $\partial^{|\lambda|} / \partial x_{1}^{\lambda_{1}} \cdots \partial x_{n}^{\lambda_{n}}, \gamma-$ произвольное число, $G$ - ограниченная область в $\mathbb{R}^{n}(n \geqslant 2)$ с границей $\partial G$ класса $C^{1}, r(x, \partial G)$ - расстояние от точки $x$ до $\partial G$.

Пусть $D$ - любое открытое множество в $\mathbb{R}^{n}$, компактно вложенное в $G$, и $G_{0}$ - некоторая область с гладкой границей, содержащая $\bar{D}$ и содержащаяся в $G$ и такая, что

$$
\min _{x \in D} r\left(x, \partial G_{0}\right)>\frac{1}{2} \min _{x \in \bar{D}} r(x, \partial G) .
$$

Полагая $\gamma=0$ в неравенстве (7), имеем

$$
\left\|\left(D^{\lambda} p_{N}(x)\right)\left(\left(\sqrt{r\left(x, \partial G_{0}\right)}+N^{-1}\right)\right)^{|\lambda|}\right\|_{p, G_{0}} \leqslant C\left(\lambda, n, G_{0}\right) N^{|\lambda|}\left\|p_{N}(x)\right\|_{p, G_{0}} .
$$

Учитьвая выбор области $G_{0}$, отсюда получим оценку

$$
\left\|D^{\lambda} p_{N}\right\|_{p, D} \leqslant C(\lambda, n, G, D) N^{|\lambda|}\left\|p_{N}\right\|_{p, G} .
$$

Продолжение доказательства до его завершения слово в слово совпадает с продолжением доказательства теоремы 4 работы автора [12, с. 73-76], которая слабее, чем второе вложение доказьваемой теоремы. Это доказательство аналогично доказательству соответствуюшей обратной теоремы для наилучших приближений целыми функциями экспоненциального сферического типа, приведенного на с. 259-262 монографии [1]. Теорема доказана.

\section{§ 2. Доказательство предложения}

2.1. Сначала докажем следующую лемму.

Лемма. Функиия $f(x)=\sqrt{1-x_{1}^{2}}$ не принадлежит пространству $B_{p, q}^{\alpha}(G)((-1$, 1) ${ }^{n}$ ) ни при каких значениях параметров $p, q, \alpha$, удовлетворяющих следующим условиям: $2 / 3<p \leqslant \infty, 0<q \leqslant \infty, \alpha>1 / 2+1 / p$.

ДокАЗАТЕЛЬСТво ЛЕмМЫ. Пусть $\delta$-любое достаточно малоеположительное число. Тогда при $e_{0}=(0,1, \ldots, 0)$ имеем

$$
\begin{aligned}
\omega_{2}(f, \delta)_{p,(-1,1)^{n}}= & \sup _{\substack{e \in \mathbb{R}^{n} \\
\|e\|=1}} \sup _{\substack{\| \leqslant \delta \\
\geqslant}}\left\|\sup ^{(2)}(\varepsilon e) f\right\|_{p,(-1,1)_{2 \varepsilon e}^{n}}\left\|\Delta^{(2)}\left(\varepsilon e_{0}\right) f\right\|_{p,(-1,1-2 \varepsilon) \times(-1,1)^{n-1}} \\
= & \sup _{0 \leqslant \varepsilon \leqslant \delta}\left\{\int_{(-1,1)^{n-1}} d x_{2} \ldots d x_{n} \int_{-1}^{1-2 \varepsilon} \mid \sqrt{1-\left(x_{1}+2 \varepsilon\right)^{2}}\right. \\
& \left.-2 \sqrt{1-\left(x_{1}+\varepsilon\right)^{2}}+\left.\sqrt{1-x_{1}^{2}}\right|^{p} d x_{1}\right\}^{1 / p} .
\end{aligned}
$$

Применяя теорему Лагранжа о конечном приращении, получим

$$
\begin{aligned}
I & :=\int_{-1}^{1-2 \varepsilon}\left|\sqrt{1-\left(x_{1}+2 \varepsilon\right)^{2}}-2 \sqrt{1-\left(x_{1}+\varepsilon\right)^{2}}+\sqrt{1-x_{1}^{2}}\right|^{p} d x_{1} \\
& \geqslant \varepsilon^{p} \int_{0}^{1-2 \varepsilon}\left|\frac{x_{1}+\varepsilon+\theta}{\sqrt{1-\left(x_{1}+\varepsilon+\theta\right)^{2}}}-\frac{x_{1}+\theta_{1}}{\sqrt{1-\left(x_{1}+\theta_{1}\right)^{2}}}\right|^{p} d x_{1},
\end{aligned}
$$


где $0<\theta=\theta\left(x_{1}\right), \theta_{1}=\theta_{1}\left(x_{1}\right)<\varepsilon$ для всех $x_{1} \in[-1,1-2 \varepsilon]$, причем из вьпуклости кверху и монотонности убывания функции $\sqrt{1-x_{1}^{2}}$ следует, что $\theta_{1}\left(x_{1}\right) \leqslant \theta\left(x_{1}\right)$ при всех $x_{1} \in[0,1-2 \varepsilon]$.

Далее оценим снизу при всех $x_{1} \in[0,1-2 \varepsilon]$ и всех достаточно малых $\varepsilon$ модуль разности

$$
\begin{aligned}
& \left|\frac{x_{1}+\varepsilon+\theta}{\sqrt{1-\left(x_{1}+\varepsilon+\theta\right)^{2}}}-\frac{x_{1}+\theta_{1}}{\sqrt{1-\left(x_{1}+\theta_{1}\right)^{2}}}\right| \\
& \geqslant \frac{x_{1}\left[\sqrt{1-\left(x_{1}+\theta_{1}\right)^{2}}-\sqrt{1-\left(x_{1}+\varepsilon+\theta\right)^{2}}\right]+\left(\varepsilon+\theta-\theta_{1}\right) \sqrt{1-\left(x_{1}+\varepsilon+\theta\right)^{2}}}{\sqrt{1-\left(x_{1}+\theta_{1}\right)^{2}} \sqrt{1-\left(x_{1}+\varepsilon+\theta\right)^{2}}} \\
& \quad \geqslant \frac{\left(\varepsilon+\theta-\theta_{1}\right)\left(1-2 x_{1} \varepsilon-2 x_{1} \theta-2 \varepsilon \theta\right)}{\left[1-\left(x_{1}+\theta_{1}\right)^{2}\right]^{3 / 2}} \geqslant \frac{\varepsilon}{2\left(1-x_{1}^{2}\right)^{3 / 2}} .
\end{aligned}
$$

Отсюда и из (9) получаем

$$
\begin{aligned}
I & \geqslant \frac{\varepsilon^{2 p}}{2^{p}} \int_{0}^{1-2 \varepsilon} \frac{d x_{1}}{\left(1-x_{1}^{2}\right)^{3 p / 2}} \geqslant \frac{\varepsilon^{2 p}}{2^{p}(2-2 \varepsilon)^{3 p / 2}} \int_{0}^{1-2 \varepsilon} \frac{d x_{1}}{\left(1-x_{1}\right)^{3 p / 2}} \\
& =\frac{\varepsilon^{2 p}}{2^{p}(2-2 \varepsilon)^{3 p / 2}(3 p / 2-1)}\left[(2 \varepsilon)^{-3 p / 2+1}-1\right] .
\end{aligned}
$$

Это неравенство с учетом оценки (8) при $2 / 3<p \leqslant \infty$ и достаточно малых $\delta>0$ дает оценку

$$
\omega_{2}(f, \delta)_{p,(-1,1)^{n}} \geqslant C(p, n) \delta^{1 / 2+1 / p} .
$$

Неравенство (10) показывает, что при $2 / 3<p \leqslant \infty$ и $\alpha>1 / 2+1 / p f \notin B_{p, \infty}^{\alpha}\left((-1,1)^{n}\right)$. Отсюда следует утверждение леммы.

2.2. Пусть $f(x)=\sqrt{1-x_{1}^{2}}$. Так как для любых $x^{\prime}, x^{\prime \prime} \in[-1,1]^{n}\left|f\left(x^{\prime}\right)-f\left(x^{\prime \prime}\right)\right| \leqslant$ $\left|\left(x_{1}^{\prime}\right)^{2}-\left(x_{1}^{\prime \prime}\right)^{2}\right|^{1 / 2}$, то для любого $\delta \geqslant 0$ имеет место неравенство $\omega(f, \delta) \leqslant \sqrt{2} \delta^{1 / 2}$. Легко заметить, что $f$ выпукла на кубе $[-1,1]^{n}$. Дальше нам нужна

ТЕОРема В. Пусть $f$ непрерывна и выпукла на отрезке $[-1,1]$,

$$
\max \{f(x): x \in[-1,1]\}=1, \quad \min \{f(x): x \in[-1,1]\}=0
$$

Тогда при $1 \leqslant p<\infty, N=1,2, \ldots$ имеет место оценка

$$
E_{N}(f,[-1,1])_{p} \leqslant C N^{-2 / p} \omega\left(f, N^{-1+1 / p}\right)_{\infty,[-1,1]} .
$$

При $p=1$ этот результат получен Ивановым [13], а при $1<p<\infty$ - Стояновой [14].

Из этой теоремы следует, что при $0<p<\infty, p_{1}=\max \{1, p\}, n \in \mathbb{N}$ и $N \rightarrow \infty$ для функции $f(x)=\sqrt{1-x_{1}^{2}}$ справедлива оценка

$$
E_{N}\left(f,[-1,1]^{n}\right)_{p}=O\left(N^{-3 /\left(2 p_{1}\right)-1 / 2}\right) .
$$

Отсюда имеем

$$
f \in E_{p, q}^{3 /\left(2 p_{1}\right)+1 / 2-\varepsilon}\left((-1,1)^{n}\right)
$$


для любых $0<\varepsilon<3 /\left(2 p_{1}\right)+1 / 2$ и $0<q \leqslant \infty$ (при $q=\infty$ здесь можно положить $\varepsilon=0)$. Из (11) и леммы вытекает утверждение а) предложения.

Как известно, для рассматриваемой функции $f(x)=\sqrt{1-x_{1}^{2}}$ вьполнено неравенство (см. $[6$, с. 91])

$$
E_{N}(f,[-1,1])_{\infty} \leqslant \frac{\pi}{2 N}, \quad N=1,2, \ldots
$$

Отсюда при $N \Rightarrow \infty$ получаем, что $E_{N}\left(f,[-1,1]^{n}\right)_{\infty}=O\left(N^{-1}\right)$. Следовательно, $f \in E_{\infty, q}^{1-\varepsilon}\left((-1,1)^{n}\right)$ для любых $0<\varepsilon<1,0<q \leqslant \infty$ (при $q=\infty$ здесь можно брать $\varepsilon=0)$. Отсюда и из леммы получаем утверждение б) предложения. Предложение доказано.

\section{СПИСОК ЦИТИРОВАННОЙ ЛИТЕРАТУРЫ}

[1] Никольский С. М. Приближение функций многих переменных и теоремы вложения. М.: Наука, 1969.

[2] Берг Й., Лефстрем Й. Интерполяционные пространства. Введение. М.: Мир, 1980.

[3] Jackson D. Über die Genauigkeit der Annäherung stetiger Funktion durch ganze rationale Funktionen gegebenen Grades und trigonometrische Summen gegebener Ordnung: Dis. Göttingen, 1911.

[4] Бернштейн С. Н. О наилучшем приближении непрерьвных функций посредством многочленов данной степени. Собр. соч. Т. 1. М.: Изд-во АН СССР, 1952.

[5] Valloe Poussin Ch. J. De la lecons sur l'approximation des functions d'une variable réelle. Paris: Gauthier-Villars, 1919.

[6] Даугавет И. К. Введение в теорию приближения функций. Л.: Изд-во Ленинградского ун-та, 1977.

[7] Никольский С. М. Некоторые вопросы приближения функций многих переменных // Конструк. теор. ф-й. Тр. Междунар. конф. (Варна, 1970). София: Изд-во БАН, 1972. С. 81-83.

[8] Коновалов В.Н. Приближение многочленами функций многих переменных с сохранением дифференциально-разностных свойств // Укр. матем. ж. 1984. Т. 36. № 2. С. 154-159.

[9] Брудный Ю. А. Об одной аппроксимационной лемме и ее применениях // Теория функций, функцион. анализ и их прилож. 1972. №15. С. 180-189.

[10] Брудный Ю. А. Теорема продолжения для одного семейства функциональных пространств // Зап. научн. сем. ЛОМИ. 1976. Т. 56. С. 170-173.

[11] Никольский С. М. Об одном методе покрытия области и неравенства для многочленов от многих переменных // Mathematica. 1966. V. 8. P. 345-356.

[12] Хатамов А. Прямые и обратные теоремы теории полиномиальных аппроксимаций функций многих переменных // Узб. матем. ж. 1993. № 4. С. 66-76.

[13] Ivanov K.G. Approximation of a convex function by means of polinomials and polygons in $L$-metric // Approximation and Function Spaces. Proc. Conf. (Gdansk, 1979). Warszawa, 1981. P. 287-293.

[14] Stojanova M.P. Approximation of a convex function by algebraic polynomials in $L_{p}[a, b]$ $(1<p<\infty) / /$ Serdica Bulg. Math. Publ. 1985. V. 11. P. 392-397. 